\title{
Persistenza e cambiamento nei Viceré di Federico De Roberto ${ }^{1}$
}

Plus ça change, plus c'est la même chose.

Il proverbio francese, secondo cui più si cambia e più le cose restano come sono, definisce sinteticamente il rapporto, impostato nei Viceré di Federico De Roberto, tra progresso storico e reazione, o, per usare termini politicamente neutri e in forza di questo più aderenti all'ideologia del nostro autore, la dinamica che unisce persistenza e cambiamento. Implicitamente, inoltre, mette in risalto un punto importante e quasi paradossale di siffatta relazione: vale a dire che persistenza e cambiamento, nell'evoluzione del singolo e della società, procedono di pari passo, e vanno considerati insieme, malgrado abbiano, all'apparenza, natura opposta, perché la conservazione di una situazione ed il mantenimento della stabilità al suo interno, secondo i canoni darwiniani seguiti da De Roberto, richiedono continui aggiustamenti. Questo legame fra elementi tradizionalmente opposti è un esempio del principio generale su cui si basa l'organizzazione del romanzo e che informa ogni singola parte: infatti ogni evento, ogni pensiero ed ogni parola che il lettore incontra hanno un valore di definizione relativo, limitato dal confronto e dal contrasto con il loro insieme. Questa affermazione, che si può genericamente sottoscrivere per qualsiasi opera letteraria, trova un significato specifico nel romanzo di De Roberto.

Generalmente persistenza e invarianza vengono considerati come stati naturali o spontanei, da accettare come dati di fatto e quindi tali da non richiedere spiegazioni, mentre il cambiamento e la novità sono considerati un problema da spiegare o un obiettivo da raggiungere. De Roberto, nei Viceré, si propose di mostrare che i due fenomeni (rinnovamento e conservazione) sono equivalenti e complementari, e che ogni situazione storica o biografica è problematica, priva di un valore assoluto; il valore, infatti, è una variabile dipendente dal punto di vista, ossia dalle parole che si usano per definire e dare forma alla realtà. Tutte le visioni della realtà, anzi, sono individuabili come una costruzione linguistica, regolata da precisi richiami semantici e completata dall'accostamento di prospettive differenti.

Nel romanzo, l'osservazione dei meccanismi del progresso nella storia della società e dell'individuo viene condotta dall'angolatura di un unico gruppo sociale, quello dei viceré, gli Uzeda (una famiglia di nobili siciliani ancora poten- 
ti), ${ }^{2}$ seguiti nell'arco di tempo che va dal 1855 al 1882 . Sebbene compaiano più volte, anche fra i personaggi minori, esempi del mutamento sociale in atto presso tutte le classi, possiamo rilevare che soltanto i personaggi appartenenti alla famiglia Uzeda sono oggetto di reale evoluzione nel corso dell'opera. È quindi tale evoluzione, come reazione a mutate condizioni storiche, ciò che interessa l'autore, al punto che l'inizio e la conclusione del romanzo recano indizi i quali ci rimandano, in forma significativa, al rapporto fra persistenza e cambiamento. Fin dal primo capoverso, i segni di quel passato contraddistinto dalla potenza degli Uzeda vengono a scontrarsi con le "novita" dei tempi:

Giuseppe, dinanzi al portone, trastullava il suo bambino, cullandolo sulle braccia, mostrandogli lo scudo marmoreo infisso al sommo dell'arco, la rastrelliera inchiodata sul muro del vestibolo dove, ai tempi antichi, i lanzi del principe appendevano le alabarde [...]. $(1.1 .1: 413)^{3}$

La continuità fra passato, presente e futuro, raffigurata in questo bozzetto di vita quotidiana, risulterà scossa una prima volta dalla "novità" della morte della principessa Teresa ("Don Salvatore?. . . Che c'è? . . Che novità? . .." 1.1.1: 413); e la valenza simbolica degli elementi di scena introdotti nell'esordio (il portone del palazzo ed il suo custode, lo stemma, la rastrelliera) ritornerà a segnare lo sviluppo dell'intreccio nei punti nodali. La prima volta ciò si verifica nel momento in cui il principe Giacomo raggiunge l'apice della potenza, nell'organismo familiare e sociale, alla conclusione felice di tutti $\mathbf{i}$ suoi intrighi, $\mathrm{i}$ quali, nondimeno, avendo per obiettivo la restaurazione del patrimonio feudale della famiglia Uzeda, additano il traguardo e, ad un tempo, il limite del concetto tradizionale di potere, nel cui ambito terre e denaro sono equiparabili come oggetti inerti da tesaurizzare anziché divenire base attiva del commercio:

agli occhi del principe, la vecchiaia dei mobili e delle livree era come un altro titolo di nobiltà; e se tutti tenevano adesso il guardaportone mentre vent'anni addietro c'era in città solo quello di Casa Uzeda, chi aveva nel vestibolo la rastrelliera? . . . (2.7.2: 852)

Nel rispetto dell'identificazione fra nobiltà e apparenze, postulata in questo ragionamento del principe (dove il rispetto del canone impersonale del verismo trapassa in ammiccamento ironico dell'autore), quei riferimenti all'ingresso del palazzo degli Uzeda torneranno fuori ancora quando occorrerà ribadire l'aspetto puramente formale della trasformazione politica degli Uzeda, la quale si realizza compiutamente con la carriera politica del principino Consalvo, prima eletto sindaco di Catania e quindi candidato vittorioso alla carica di deputato del regno d'Italia. Il primo evento fa rinascere nel personaggio l'albagìa della stirpe nobiliare di cui il guardaportone è ancora uno degli emblemi distintivi (a dispetto dell'opinione di Giacomo riportata in modo indiretto nella citazione precedente): ${ }^{4}$

Sapeva che la grande popolarità della sua casata dipendeva dal fasto esteriore, dalle 
livree fiammanti, dalle carrozze rilucenti, dal guardaportone maestoso [...]. (3.5.1: 971)

Più avanti, la ripresa quasi letterale della descrizione contenuta nell'incipit del romanzo ("mostrandogli lo scudo marmoreo infisso al sommo dell'arco, la rastrelliera inchiodata sul muro del vestibolo" 1.1.1: 413; "vergognoso del grande stemma infisso sull'arco del portone, della rastrelliera del vestibolo" 3.9.1: 1065) si accompagna alla consapevolezza che $\mathrm{i}$ tempi nuovi della democrazia hanno generato l'esigenza e la moda di altri orpelli, come l'ostentazione dell'umanitarismo, un'infarinatura di nozioni politiche e filosofiche, la teatrale esibizione delle qualità oratorie: ${ }^{5}$

pareva volersi scusare del suo titolo e delle sue ricchezze, quasi vergognoso del grande stemma infisso sull'arco del portone, della rastrelliera del vestibolo, dei ritratti degli avi, come d'altrettante macchie, d'altrettanti attestati d'indegnità. (3.9.1: 1065)

Ma l'esemplarità dell'esordio e della sua ambientazione acquisteranno un risalto drammatico e paradossale nell'episodio dell'incontro fra don Eugenio, zio del principe Giacomo, e Pasqualino, da lunga data cocchiere e servitore di casa Uzeda:

Fatto un gesto d'indignazione, Pasqualino prese un'altra seggiola nel bugigattolo, e sedette accanto al cavaliere [. . . ]. Al fresco del vestibolo la conversazione si prolungava: padrone e servo discorrevano intimamente, da pari a pari, mescolando il fumo della pipa e del sigaro; anzi, quantunque Pasqualino non fosse elegante come un tempo, pure sembrava il padrone, e don Eugenio il creato. Il guardaportone, tra scandalizzato ed invidioso della confidenza [. . . ] spasseggiava dignitosamente dinanzi all'entrata [...]. (3.1.1: 896-97)

In questa scena, consapevole allegoria e insieme caricatura dei contenuti della propaganda politica coeva (l'incontro fra la vecchia classe dominante ed il popolo che si affaccia alla scena della storia "da pari a pari"), ${ }^{6}$ il vestibolo del palazzo Francalanza è custodito da un guardaportone che, sebbene assuma qui spessore di personaggio vivo (contro la riduzione a parte della cornice che si era verificata negli altri esempi), è chiamato una volta di più ad assumere la funzione di nume tutelare del privilegio di casta (e il senso della trasgressione in atto di fronte a lui traspare tanto nel suo atteggiamento, "tra scandalizzato ed invidioso", quanto, e con icastica efficacia, nel suo "spasseggiare dignitosamente dinanzi all'entrata", quasi a ripristinare, amplificando la sua presenza, l'onore violato di casa Uzeda, come ad arginare la portata dell'offesa contro le convenzioni sociali). Il vestibolo, per di più, diventa il luogo dove profeticamente si realizza quell'affratellamento tra il nobile ed il popolano auspicato da Consalvo nei suoi discorsi demagogici, la cui retorica affettazione di egualitarismo verrà volgarizzata dai suoi detrattori in termini che paiono una chiosa al quadro edificante dipinto dall'amichevole conversazione di Pasqualino e don Eugenio:

a sentirlo, tutti gli uomini erano fratelli, fatti per sedersi sopra una stessa panca ... (3.5.1: 972) 
Gli esempi che abbiamo mostrato finora, identificati da un processo di ipostatizzazione di tematiche sociali al quale l'incipit dà l'abbrivio, sono già sufficienti a delineare il particolare tipo di rapporto che si instaura fra gli elementi del sistema socio-politico descritto nei Viceré.

Se il romanzo si apre con il guardaportone che indica al figlio i segni della potenza e della nobiltà della famiglia Uzeda, e se a distanza di anni si riconosce ancora in quei segni più esterni la distinzione che proviene dal sangue nobile $\mathrm{e}$ dall'antichità dei titoli, la vera chiave di volta del motivo è la constatazione che, pur rinnovandosi i tempi a causa degli esiti del Risorgimento, resta intatta negli aristocratici come Consalvo la consapevolezza del prestigio che deriva proprio dal "fasto esteriore" (3.5.1: 971); e alle proferte di umiltà e di democrazia del principino, rammentate poc'anzi, fa seguito un'esplicita valutazione della portata di simili affermazioni, un'osservazione del narratore tanto più sferzante quanto più la veste è dimessa e l'intonazione neutra: "Ma egli faceva questo a tempo e luogo" (3.9.1: 1065).

Con un procedimento strutturalmente simile, solo diverso e più ricco di umori nell'esecuzione formale, la scena della conversazione tra Pasqualino e don Eugenio muove dal riconoscimento dell'assenza di ogni formalità (“da pari a pari”), per introdurre con gradazione ironica il commento paradossale che amplifica il concetto di uguaglianza appena accennato e ne mina il fondamento obiettivo, già fragile, rovesciando i rapporti nella scala sociale fra i due personaggi: "anzi, [...] Pasqualino [. . . ] sembrava il padrone, e don Eugenio il creato". Stabilite simili premesse, la reazione del guardaportone, più che ribadire la misura dell'impressione che fatti del genere suscitano nel popolo, induce a riconsiderare lo status sociale delle figure in campo, tant'è vero che il paragrafo si chiude con una battuta dello stesso guardaportone, il quale, richiesto di indicare chi sia la persona che parla con Pasqualino, accenna dubbioso all'evidente differenza fra il padrone, il principe Giacomo, e don Eugenio: "Uno zio del principe, dice!" (3.1.1: 897).

Mescolato agli intenti satirici, v'è qui l'enunciato che la rivoluzione in atto nella storia, in effetti, determina il tramontare solo di una certa nobiltà di parata, alla quale restano ormai soltanto i titoli, ${ }^{7}$ mentre potere e ricchezza salvaguardano intatta la posizione dei principi di Francalanza, così abili da convertire in opportunità di profitto quello stesso fenomeno, sfruttando la decadenza dei membri meno dotati ed attivi della famiglia (suor Crocifissa, don Eugenio, Ferdinando). Nel complesso, l'attenzione della società verso gli attributi esteriori del potere, vecchio e nuovo, maschera la realtà, cioè il fatto che resta invariata nel tempo la distanza che separa il popolo dai potenti.

Il modello stilistico ed ideologico appena tracciato fa nuova mostra di sé in una descrizione che interviene, durante la campagna elettorale di Consalvo, nel momento in cui si vogliono spiegare i nuovi rapporti che si stabiliscono fra il principino, desideroso di ostentare modi democratici coerenti con la sua nuova immagine, e Baldassarre, che in passato aveva ricoperto la mansione di maestro 
di casa a palazzo Francalanza ed ora si incarica di curare l'azione di propaganda del candidato:

il padrone e il servo erano scomparsi, sedevano a fianco alla stessa tavola, il principe passava la carta e la penna all'antico creato, si davano del lei come due diplomatici stipulanti un trattato. (3.9.2: 1073)

Il meccanismo è analogo a quello dell'esempio precedente: la similitudine, che, seguendo il filo logico dello sviluppo del discorso. parrebbe diretta a rinforzare l'idea suggerita dalla scena, si tramuta in un appunto caricaturale atto a far brillare comicamente la natura esteriore e teatrale delle novità politiche postunitarie, grazie all'impiego magistrale di una similitudine ("come due diplomatici") la quale, nel crescendo pomposo ed ironico della specificazione che ne prolunga la nota già quasi stonata ("stipulanti un trattato"), disvela un'enfasi volutamente inadeguata (e inconsistente) rispetto al compito che le è affidato, di bilanciare quella distanza sociale due volte sottolineata nel brano ("il padrone e il servo"; "il principe", "l'antico creato"). Tra la collaborazione di Baldassarre all'allestimento dei funerali della principessa Teresa, in apertura di romanzo (nell'anno 1855), e la sua attività di sostenitore politico del principino nelle elezioni del 1882 , al termine della storia, la differenza non oltrepassa il mondo delle pure idealità, confinata nella sfera nominale, che testimonia, nell'uso delle formule allocutive, una supposta familiarità fomentata dalla nuova era di democrazia, in cui i principi scendono dal loro piedistallo ("«Signor principe» non gli dava più, per democrazia, dell'Eccellenza, «la nostra società ha fatto iscrivere una cinquantina di elettori [. . . ]»" 3.9.2: 1073), ed i servi sono promossi a dignità di nuovo conio, purché abbiano le qualità che si richiedono, ossia "la gravità" ed un aspetto "più serio e decorativo di tanti altri":

egli si guardava intorno ed ascoltava con la gravità dell'antico maestro di casa, era più serio e decorativo di tanti altri; un sindaco di provincia che gli stava a fianco gli disse: «Da noi la riuscita è assicurata. E qui, professore, come vanno le cose?» «Eccellente!» fece Baldassarre, scrollando il capo. (3.9.2: 1073)

Anzi, se il cambiamento annunciato dal "soffio dei nuovi tempi" (1.3.5: 497; 1.3.8: 518) sortisce un esito reale, sarà quello di produrre una situazione di vantaggio per quei discendenti degli antichi viceré che sanno mettere a frutto l'eredità dei padri. All'atto pratico, e per culmine di ironia derivante dall'interpretazione letterale della vicenda, si assiste nel romanzo all'avanzamento dei potenti a spese del popolo; i nuovi privilegi di Baldassarre, divenuto "libero cittadino", si traducono negli oneri di una servitù volontaria:

andava e veniva, sudato, sbuffante, come ventotto anni addietro, quando ordinava l'aristocratico cerimoniale dei funerali della vecchia principessa. Ma allora egli era servo stipendiato, e adesso libero cittadino che interveniva a un metingo democrati$\mathrm{co}$, e che prestava il suo appoggio al principe non per quattrini ma per un'idea. (3.9.3: 1078) 
Così, in un'altra occasione, il rispetto del significato letterale assegnato ad una frase pronunciata a mo' di slogan propagandistico da Consalvo, durante un discorso elettorale, consente di stravolgere lo spirito democratico del motto e porta alla luce non solo la cinica simulazione del personaggio, ma anche lo scarso peso di certe dichiarazioni di principio tanto in voga:

"[. . .] Milioni e milioni d'uomini liberi possono volontariamente riconoscersi e vantarsi sudditi di un uomo come loro? Io non ho nessun padrone!». E in questo era sincero, perché avrebbe voluto esser egli stesso padrone degli altri. $(3.6 .1: 997)^{8}$

I casi che abbiamo fin qui riportato, in sostanza, chiariscono il ricorso dell'autore ad artifici stilistici costanti; in concreto, la smentita dei termini "parità" e "uguaglianza" (come di altre parole d'ordine dell'epoca a lui contemporanea) è compiuta con l'uso provocatorio di notazioni che sfiorano la dimensione dell'iperbole grazie ad un processo di accumulazione, sul piano semantico e formale, il quale prende le mosse di solito da un'osservazione ambigua o velata e, per via di precisazione e specificazione, stabilisce un crescendo che immette nel discorso dissonanze così pronunciate da confermare la fondatezza di quel sospetto di falsità insinuato prima, in tono minore, nelle maglie di una prosa che mimava il rispetto delle convenzioni stabilite, e si scuote poi dal proprio prevedibile fluire con un guizzo, l'umorismo grottesco marcato dall'uso di tonalità stridenti, eccessivamente alte o basse: il nobile abbassato a "straccione" (3.1.1: 897); il servo elevato al rango di "diplomatico stipulante un trattato".

Nell'incontro fra don Eugenio e Pasqualino l'accenno all'intimità della conversazione ("padrone e servo discorrevano intimamente") trapassa in una specificazione ("da pari a pari") non opaca a quella significazione ironica che acquista piena luce nella resa di tale uguaglianza in un dettaglio di basso realismo ("mescolando il fumo della pipa e del sigaro"), per toccare il suo vertice nella precisazione finale ("anzi, [. . .] Pasqualino sembrava il padrone, e don Eugenio il creato" 3.1.1: 897). Allo stesso modo, nei commenti che irridono alle ansie democratiche del principino, il peso del concetto di universale fratellanza a cui si allude ("a sentirlo, tutti gli uomini erano fratelli") si misura sulla futilità della sua rappresentazione concreta ("fatti per sedersi sopra una stessa panca"). Altrettanto ingannevole, rispetto alla direzione che prenderà il discorso, appare l'impressione che dà il ritratto di Consalvo e Baldassarre ("sedevano a fianco alla stessa tavola"), rinforzata apparentemente dall'attitudine di umiltà e di fraternità del principe ("passava la carta e la penna all'antico creato", "si davano del lei"); l'esito della scena, del tutto fuori tono, di una solennità sproporzionata all'occasione che la suscita ("come due diplomatici stipulanti un trattato"), ha l'effetto di rimettere in discussione l'idillica armonia del quadretto edificante che l'autore veniva strutturando, sul fondamento di una premessa dubbia ("il padrone e il servo erano scomparsi").

Per completare l'esame di questo modulo stilistico nella serie di esempi forniti, possiamo indicare lo stesso movimento narrativo nel paragone istituito fra il 
tempo in cui Baldassarre sovrintendeva al cerimoniale del funerale della principessa Teresa e il presente, che lo vede a fianco del principe Consalvo nella campagna elettorale. Anche qui il tono asseverativo dell'antitesi moraleggiante stabilita fra le qualifiche di "servo stipendiato" e di "libero cittadino", mentre lascia pensare che il narratore possa indirizzarsi, nell'aggiunta conclusiva, verso un'ulteriore conferma del passaggio di qualità occorso, sfocia in un'affermazione che di fatto nega il miglioramento ipotizzato ("prestava il suo appoggio al principe non per quattrini ma per un'idea") per rinforzare al contrario i motivi della fatica e dell'attività servile espletata in nome di quell'ideale democratico che nega il compenso prima dovuto, ma non esime dall'omaggio, reiterato sotto altre forme, alla nobiltà, ricollegandosi perciò alle memorie del funerale con cui tutto il discorso si era iniziato ("andava e veniva, sudato, sbuffante, come ventotto anni addietro, quando ordinava l'aristocratico cerimoniale dei funerali della vecchia principessa")."

In sostanza, l'opera di demistificazione della storia ufficiale perseguita da De Roberto nei Viceré si realizza per il tramite di una sottile ironia, capillarmente infusa nel romanzo, intesa a dimostrare il valore meramente retorico delle agiografie risorgimentali che circolavano nello scenario politico italiano sul finire del secolo. La via che egli seguì fu in primo luogo l'adozione della struttura narrativa di tipo verista, mirante a restituire luci ed ombre dell'epoca con forza polemica integra (appena venata di psicologismo, ma ben lontana dall'approdo, oscillante fra nichilismo ed ottimismo consolatorio, dell'ultimo e più stanco romanzo del ciclo, L'Imperio). Tuttavia ciò che salvò l'autore dalle debolezze insite in un genere ormai prossimo ad esaurire la propria vitalità artistica, e lo sottrasse di fatto da quel "tramonto del verismo" in cui tanta parte della bibliografia critica si ostina a confinarlo, resta l'appropriazione, che egli tentò, del vocabolario e dei motivi di quell'historia postunitaria, per misurame la credibilità sul paragone di un contesto storicamente verisimile e per rivelarne l'intrinseca debolezza con un'ironia che attinge una carica dirompente dal contrasto con lo sfondo, superficialmente realistico ed oggettivo eppure già variegato e mosso da forze nuove.

Infine, alle tentazioni del compromesso ed alle speranze di pacifica convivenza coltivate sul terreno del progresso scientifico di marca positivista, De Roberto volle contrapporre, con un pessimismo più iroso che tragico, ${ }^{10}$ la resistenza opposta ad ogni cambiamento dalle stesse leggi di natura definite da Darwin. La storia del testo dei Viceré (ricostruibile sulla scorta delle notizie riportate nelle lettere di De Roberto al giovane amico Ferdinando Di Giorgi) testimonia in modo eloquente tale proposito. In una lettera che reca l'indicazione "Milano, 16 luglio 1891" " De Roberto afferma:

Quando sarò tornato a casa, attaccherò i Viceré (te ne ho parlato?). Ho smessa l'idea di scrivere la Realtà (almeno per ora) e vo' preparare questi Viceré, che sarà un romanzo . . . come? Non lo so ancora. Ti posso dire soltanto l'idea: la storia d'una gran famiglia, la quale deve essere composta di quattordici o quindici tipi, tra maschi e femmine, uno più forte e stravagante dell'altro. Il primo titolo era Vecchia 
razza: ciò ti dimostri l'intenzione ultima, che dovrebbe essere il decadimento fisico e morale d'una stirpe esausta. Vedremo! (Navarria 273)

Sia il primo titolo dell'opera, Vecchia razza, che quello definitivo dimostrano come l'originaria sollecitazione a mettere mano al romanzo provenisse dall'influenza delle teorie naturaliste sull'impronta determinata dalla race nell'individuo. Ma fin da questo primo accenno salta agli occhi un paradossale accostamento fra la "forza" attribuita ai personaggi e l'intenzione proclamata di metterne in luce il "decadimento fisico e morale". Solo la classificazione dei personaggi come "tipi" ed il riferimento alla loro "stravaganza" fanno presagire le modalità di una coniugazione teorica fra la "forza" e la "decadenza": le premesse del libro stanno tutte nella posizione singolare ed anomala-di potenza-che i viceré (rappresentanti di una più ristretta categoria di nobili) ${ }^{12}$ occupano e mantengono nell'ambito di una società messa in subbuglio dagli eventi del Risorgimento, che vede affermarsi la partecipazione alla vita politica del ceto borghese e, in minor misura, perfino del popolo.

Se "stirpe esausta" prelude dunque alla scomparsa di una casta sopravvissuta al feudalesimo, una sorta di fossile sociale, la "stravaganza" (da assumere, in chiave darwiniana, come variabilità) e la "forza" presuppongono la possibilità di un'evoluzione, che implica certamente la fine di una "stirpe", ma come premessa della sua trasformazione. Del resto lo stesso titolo scelto, I Viceré, evocando il potere e l'elevata condizione sociale dei protagonisti, si oppone in modo evidente alla negatività del titolo primitivo, Vecchia razza, a conferma dello sviluppo attuatosi nel nucleo concettuale che regolava la formazione del romanzo.

In pochi mesi d'assidua applicazione De Roberto si dedicò ai capitoli d'apertura e a quelli necessari perché risultassero delineati la scena, i nodi ed il contesto storico del romanzo. Nel settembre del 1891 i primi due capitoli sono già impostati (cfr. Navarria 283-84). Stabilito l'incipit del romanzo, l'idea che lo sorregge comincia ad essere elaborata, e in ottobre lo stato di sviluppo del libro è descritto così:

Siamo già al $7^{\circ}$ capitolo, e col $10^{\circ}$ si chiuderà la prima parte. Ce ne sarà una seconda lunga press'a poco quanto la prima, e poi basta. [. . .] Mi sono messo al lavoro, al solito, senza piano, senza sapere dove andare a sbattere le corna, con un germe di idea; a poco a poco questo s'è venuto sviluppando, e adesso mi pare di vederci chiaro. I Viceré doveva essere la storia d'una famiglia di nobili prepotenti e stravaganti, ma quanti dovevano essere i membri di questa famiglia? In quale epoca doveva svolgersi questa storia? Quali avvenimenti dovevano formarla? Non ne sapevo nulla, e scrissi così tre capitoli. (Navarria 285-86)

Dell'idea primitiva restano saldi, nel primo abbozzarsi delle vicende, la centralità della famiglia dei viceré e la sua caratterizzazione mediante la "stravaganza": la forza di questa famiglia adesso è connotata, con una sensibile accentuazione, dal termine "prepotenti"; nessun cenno più alla decadenza. Si consolida quindi il vincolo già postulato tra singolarità di comportamenti e attitudine alla 
prevaricazione. Inoltre si aggiunge l'esigenza di stabilire una correlazione precisa tra gli avvenimenti, in particolare quelli storici, e gli attributi della famiglia; ma si deve osservare che la vicenda nasce proprio intorno a questi attributi, mentre soltanto in un secondo momento si avverte l'esigenza di precisarne i contorni storici, come se essi rappresentassero il mero pretesto per esporre un assunto valido in ogni tempo.

La struttura dei Viceré, quindi, appare costruita fin dall'inizio intorno a due blocchi narrativi: la storia della famiglia Uzeda, movimentata dai fatti conseguenti al testamento della principessa, e la trasformazione della società italiana sotto la spinta degli eventi risorgimentali. Nella redazione definitiva del romanzo, questi due blocchi, ravvicinati dal punto di vista della concezione e della materia, vennero distanziati con l'interposizione di alcuni capitoli che descrivono le vicissitudini di tre generazioni di Uzeda (cap. 1.3), la tormentosa esperienza matrimoniale della moglie di Raimondo Uzeda, Matilde (capp. 1.4 e 1.5), le gloriose memorie della famiglia dei viceré (cap. 1.5) e la poco edificante storia del convento e dei monaci di San Nicola (cap. 1.6).

La dilatazione della misura storica così prodotta genera una rivoluzione cospicua nella natura e nelle intenzioni del testo, poiché sposta l'attenzione dall'elementare convergenza di una cronaca familiare con l'"eroica" storia nazionale, ${ }^{13}$ dal semplice incontro di realtà ugualmente prosaiche, e la indirizza da un lato verso il riconoscimento dei processi linguistici che danno forma alla realtà e sono la chiave per decodificarne l'aspetto recondito (per chiarire qualità ed entità di ogni cambiamento), dall'altro verso l'individuazione di leggi naturali valide anche nell'arco di un più lungo periodo storico.

In proposito non bisogna dimenticare come la cultura di fine Ottocento fosse influenzata da visioni antitetiche sul destino del mondo: se la teoria di Darwin affermava la continuità del progresso, sia pure non in senso assoluto, la conoscenza degli attributi e degli effetti del secondo principio della Termodinamica, formulato al principio del secolo, suggerirono a intellettuali come Morel, Lombroso e Nordau (che De Roberto ben conosceva: cfr. Di Grado) l'idea apocalittica di un graduale decadimento di tutte le cose. Evoluzione e degenerazione, la tendenza ad un ordine complesso o al massimo disordine, erano nella mentalità dell'epoca i due poli della natura, in perenne contrasto fra loro nella conformazione del mondo e della società (cfr. Arnheim); nello stesso ciclo progettato da De Roberto, mentre i Viceré si chiudono con il riproporsi-in termini diversi e secondo un meccanismo più raffinato ed elaborato—del modello politico feudale, L'Imperio sfiora la prospettiva del rifiuto della vita moderna, ipotizzata da Nordau, fino a immaginare gli strumenti e le tappe del "suicidio cosmico" di cui aveva parlato Hartmann e a cui De Roberto stesso si riferiva espressamente nell'Amore (12).

La consapevolezza della dinamica evolutiva, nei Viceré, è sottolineata al termine di ciascuna delle tre parti del romanzo, con la riproposta, in posizione strutturalmente significativa, della sorte di quei personaggi a cui pare affidata la sopravvivenza e la futura gloria degli Uzeda: il duca d'Oragua, Consalvo e Lo- 
dovico, rappresentanti il potere nei suoi diversi aspetti (temporale ed ecclesiastico). ${ }^{14} \mathrm{Al}$ termine della prima parte, quando il duca d'Oragua viene eletto deputato per la prima volta, il principe Giacomo lo indica al figlio Consalvo come modello esemplare della capacità di adattamento al mutarsi delle istituzioni politiche:

vedi lo zio come fa onore alla famiglia: quando c'erano i Viceré, i nostri erano Viceré; adesso che abbiamo il Parlamento, lo zio è deputato! ... (1.9.4: 697)

Al termine della seconda parte, in occasione del ritorno a Catania di Lodovico, nel 1870 , si traccia un profilo della sua folgorante carriera:

Egli aveva dato appena una volta all'anno notizie di sé alla famiglia, tutto intento ai doveri del suo ufficio, alla preparazione della sua fortuna che oramai era avviata. In poco più di tre anni era già segretario a Propaganda ed arcivescovo di Nicea; Pio Nono aveva molta stima di lui. (2.9.2: 883 )

Infine i Viceré si chiudono con l'elezione di Consalvo a deputato del regno, nel 1882 , e, più precisamente, con le parole che questi rivolge alla prozia, donna Ferdinanda, la quale incarna il culto del passato, delle "memorie dei fasti degli antenati" (1.3.7: 513). L'ultima frase del dialogo fra i due, che è poi la chiusa del romanzo, ("la nostra razza non è degenerata: è sempre la stessa" 3.9.4: 1103) sigla la conclusione del ciclo narrativo aperto dallo smembramento del patrimonio di casa Francalanza (previsto dalle disposizioni testamentarie della principessa Teresa), complicato dalle rivoluzioni politiche e ricondotto all'equilibrio dal primato raggiunto da Consalvo nelle prime elezioni a suffragio allargato.

Il fatto che le tre partizioni del romanzo si chiudano specularmente all'insegna di episodi e di motivi che pongono l'accento sul rinnovarsi dell'antico prestigio dei viceré acquista un valore meglio definito se osserviamo come, in tutti e tre $\mathrm{i}$ casi citati, $\mathrm{i}$ successi di alcuni personaggi fanno da contrappunto alla descrizione di altre manifestazioni, di carattere patologico, le quali, lungi dal rappresentare semplicisticamente l'invecchiamento della razza, sono un indice della sua vitalità, lasciano presagire la sua imminente evoluzione; in senso darwiniano, infatti, mostri e campioni non sono che gli estremi opposti sulla scala delle variazioni di una specie. ${ }^{15}$

Nel paragrafo che conclude la prima parte (1.9.4), la vittoria del duca si intreccia alla descrizione della gravidanza di Chiara, fino al parto di un aborto mostruoso:

dall'alvo sanguinoso veniva fuori un pezzo di carne informe, una cosa innominabile, un pesce col becco, un uccello spiumato; quel mostro senza sesso aveva un occhio solo, tre specie di zampe, ed era ancor vivo. (1.9.4: 691)

Un fenomeno di tal genere, nel contesto della teoria di Darwin, è strettamente correlato al processo evolutivo di ogni specie, come illustra, fra gli altri, un passo del capitolo quinto dell'Origine delle specie, dedicato alle leggi della variazione: 
Si può dire a buon conto che esiste una lotta costante fra la tendenza alla reversione ad uno stato meno modificato, da una parte, contemporaneamente ad una tendenza innata a variazioni di ogni genere, e, d'altra parte, la capacità della selezione continuativa, che tende a mantenere pura la razza. [. . .] Finché la selezione è in corso con rapidità, dobbiamo sempre attenderci che le strutture che si vanno modificando siano anche molto variabili. (197)

L'esaltazione di Lodovico, d'altro canto, avviene proprio nel momento in cui Ferdinando, nel disfacimento dell'organismo e neli'acuirsi della pazzia, fa mostra di alcune fissazioni maniacali, come il sospetto, la diffidenza e l'avidità, che celano, a detta dello stesso narratore, una natura 'positiva', dato che in altro tempo-si intuisce-lo avrebbero difeso dalle ruberie dei parenti. Ferdinando sconta la sua appartenenza alla razza dei Viceré con la morte, perché dalla famiglia ha ereditato i germi di una degenerazione mortale ("il sangue vecchio e impoverito dei Viceré", "le flaccide fibre"), e non conosce che una variazione tardiva e distorta di quell'istinto di prepotenza e aggressività, caratteristico della stirpe degli Uzeda ("si rivelava a un tratto dei Viceré"), che assicura la sopravvivenza nella nuova società ad alcuni dei suoi membri:

A trentanove anni egli se ne moriva: il sangue vecchio e impoverito dei Viceré si dissolveva, non nutriva più le flaccide fibre. (2.9.1: 879)

Ferdinando era impazzito del tutto. La sorda diffidenza destatasi in lui contro $\mathrm{i}$ fratelli, il secreto sospetto che non gli aveva consentito di attribuire all'affezione le loro premure fastidiose, erano cresciute di giomo in giomo e invaso talmente il suo cervello che non capiva più nessun'altra idea. Egli che per trentanove anni aveva dato prova di tanto disinteresse da meritar dalla madre il nome di Babbeo, da lasciarsi rubar da tutti, si rivelava a un tratto dei Viceré con quel sospetto buffo e pazzo, adesso che non aveva più nulla da lasciare. $(2.9 .2: 885-86)$

Non bisogna dimenticare che, nei passi citati sopra ed anche altrove, decadimento fisico e istinto di sopraffazione sono considerati tratti coerenti ed ugualmente pertinenti, come $\mathrm{i}$ due lati di una medaglia, nella rappresentazione dei $\mathrm{Vi}$ ceré; solo il sostrato darwiniano consente di giustificare ed inquadrare l'apparente antinomia. Sono illuminanti al riguardo certe generalizzazioni, fondate sulla scienza biologica dell'epoca (soprattutto Darwin) e applicate all'esame dei rapporti sociali, che leggiamo in quella ponderosa opera saggistica, L'Amore, che De Roberto pubblicò l'anno successivo al romanzo maggiore. Vi rintracciamo passi che chiariscono la gradazione di somiglianze e differenze, fra i membri della famiglia Uzeda, e giustificano casi come quello di Ferdinando:

La storia naturale dimostra che tutti gli esseri viventi [. . .] formano una specie di scala o gamma dove le modificazioni e lo sviluppo delle strutture e delle attitudini procedono per gradi e in modo tale che, se due tipi molto discosti l'uno dall'altro sono tanto diversi da parere anche indipendenti, l'esame di tutti i tipi intermedii fa scoprire per qual serie di successivi mutamenti essi si collegano. (1) 
Se pensiamo che tra la scimmia ed il genio esistono il selvaggio e l'uomo comune, vediamo che la diversità procede per gradi, quindi che vi sono gradi d'affinità. (2)

$\mathrm{Nel}$ rispetto della dinamica che siamo venuti esponendo, l'elezione di Consalvo è preceduta dalla morte del padre, ultimo rappresentante di un desiderio di potere intenso, ma limitato alla cerchia familiare e cittadina, messo in forse dalle novità dei tempi. I segni della malattia paterna, che non a caso ha carattere tumorale, sono collegati esplicitamente alla debolezza della "razza", del "sangue", che per altro corrisponde alla sopravvenuta incapacità di Giacomo di fronte all'esigenza di adattarsi al mutato contesto sociale. Sorprendentemente, di fronte alla morte del padre le parole di Consalvo esprimono la certezza che l'appartenenza alla razza degli Uzeda gli offre una prospettiva di nuova forza, anticipando la conclusione del romanzo, che vedrà immutata rispetto al passato la potenza dei viceré:

Sarebbe anch'egli morto prima del tempo, prima di conseguire il trionfo, ucciso da quei mali terribili che ammazzavano gli Uzeda giovani ancora? [. . .] Che avrebbe dato egli stesso, perché nelle proprie vene scorresse il sangue vivido e sano di un popolano? ... "Niente! . . " Il sangue povero e corrotto della vecchia razza lo faceva quel che era [...]. (3.7.5: 1039)

Risulta evidente, in brani come questo, che le malattie, la corruzione delle fibre, non pongono una seria minaccia alla sopravvivenza dei viceré e della loro autorità; semmai, si tratta del fattore discriminante che segnala, nella galleria dei tipi della famiglia, quanti sono portatori di quelle caratteristiche della stirpe che non sono più adatte al contesto sociale. Infatti le occorrenze dei termini inerenti al tema della malatia (svolto con grande ricchezza di lessico e varietà di sintomatologia) seguono l'andamento del tema dell'evoluzione, concentrandosi nella seconda e nella terza parte del romanzo. Nel loro complesso, i riferimenti alla malattia, anziché tracciare un quadro clinico della realtà, fosco e pessimistico (come gli studiosi hanno spesso inteso), sono presentati sotto forma di variazione, come il naturale complemento del principio evolutivo che si afferma in alcuni personaggi. Alle attestazioni di congenita fragilità riportate sopra ne vanno affiancate altre, che, opponendo il riaffiorare, nella famiglia Uzeda, di superstiti elementi di forza e longevità, resterebbero incomprensibili senza il supporto di una teoria biologica in grado di spiegare la coesistenza di entrambi i fenomeni all'interno di una "vecchia razza":

Tra i progenitori più lontani c'era quella mescolanza di forza e di grazia che formava la bellezza del contino [. . .]. Per una specie di reviviscenza delle vecchie cellule del nobile sangue, Raimondo rassomigliava al più puro tipo antico. (1.3.6: 502)

Teresa pareva fosse venuta fuori da una vecchia cellula intatta del puro sangue castigliano. (3.2.1: 906) 
il principe poteva vivere cent'anni, come tanti di quegli Uzeda che avevano il cuoio duro [...]. (3.2.3: 919)

il nostro sangue è impoverito; eppure ciò non impedisce a molti dei nostri di arrivare sani e vegeti all'invidiabile età di Vostra Eccellenza! . . . (3.9.4: 1102)

Condizionato dai fattori naturali descritti, il movimento della storia, il "progresso", è destinato a scontrarsi con un sistema-l'assetto sociale retaggio del feudalesimo—che tende necessariamente all'immobilità, che fa della stabilità, del "posto nel mondo" (3.7.5: 1039), l'obiettivo prioritario, e se ne serve, oltre che come fine, come mezzo per ridurre la deviazione da certe regole.

Un tempo la potenza della nostra famiglia veniva dai re; ora viene dal popolo . . . La differenza è più di nome che di fatto . . . (3.9.4: 1099)

L'affermazione di Consalvo, nel finale del libro, discende dall'intuizione che ispira il romanzo, vale a dire la possibilità di applicare alla rappresentazione della realtà la legge scientifica dell'equifinalità, che De Roberto mostra anche altrove di aver ben compreso; ${ }^{16}$ il principio, che regola il meccanismo della selezione e dell'evoluzione, secondo il quale gli stessi risultati possono avere origini diverse, giustifica il fatto paradossale che tutti gli sconvolgimenti creati dal Risorgimento non hanno provocato alcun cambiamento di rilievo nella società catanese. Il problema della difesa degli interessi sociali, brillantemente risolto dagli Uzeda, è certo uno di quelli che "ammettono più d'una soluzione" ( L'Amore 17): la sopravvivenza della razza dei viceré è procurata dalle stesse innovazioni sociali che dovevano segname il tracollo, come riconosce Consalvo nella confutazione del suo discorso pubblico rivolta alla zia Ferdinanda:

Dobbiamo farci mettere il piede sul collo anche noi? Il nostro dovere, invece di sprezzare le nuove leggi, mi pare quello di servircene! . . (3.9.4: 1101)

Nei Viceré, ciò che guida l'articolazione di dettaglio della struttura è l'organizzazione dei contenuti secondo i principi della biologia ottocentesca: il rapporto tra $\mathrm{i}$ diversi livelli linguistici del romanzo (storico-documentario, dialettale, narrativo, psicologico, ecc.) costituisce un sistema coerente, improntato al concetto che il linguaggio maschera la naturale immobilità della realtà umana e sociale, ossia la permanenza di certe leggi. In una struttura apparentemente caotica, ricca di personaggi e di episodi, si stabilisce progressivamente un ordine, che manifesta l'invarianza naturale di cui abbiamo parlato. Tutti i gesti dei viceré Uzeda rispondono alle caratteristiche del loro gruppo sociale, e sono subordinati alla sopravvivenza e alla realizzazione del loro istinto di potenza.

Al di fuori della dialettica fra progresso e regresso, in voga alla fine del secolo diciannovesimo, De Roberto ha strutturato i Viceré sulla base del principio darwiniano che così riassumeva nel secondo capitolo dell'Amore:

Il progresso, in senso assoluto, non esiste [. . .]. Nell'evoluzione non c'è né supe- 
riorità né inferiorità. [. . . ] Ad ogni grado della scala vivente non c'è né progresso rispetto al grado sottoposto, né regresso rispetto al sovrapposto, ma una relativa convenienza nella diversità. $(13-14)^{17}$

Per la comprensione della relazione dinamica che lega i piani temporali nel romanzo è essenziale intendere che le leggi immutabili della natura condizionano lo sviluppo di ogni tipo di società umana ed appaiono evidenti all'osservazione distaccata della storia:

quantunque dicessero che $\mathrm{i}$ tempi erano mutati, tutte queste cose, $\mathrm{i}$ segni visibili della ricchezza e della potenza, non avevano potuto, non potevano perdere mai, per mutar di tempi, il loro valore. (3.5.1: 971)

Quasiché, ammessa pure la possibilità d'abolire con un tratto di penna tutte le disuguaglianze sociali, esse non si sarebbero di nuovo formate il domani, essendo gli uomini naturalmente diversi, e il furbo dovendo sempre, in ogni tempo, sotto qualunque regime, mettere in mezzo il semplice, e l'audace prevenire il timido, e il forte soggiogare il debole! Nondimeno piegavasi, concedeva tutto, a parole, allo spirito dei nuovi tempi. $(3.9 .1: 1065)^{18}$

Nei Viceré l'ironica regressione dei contenuti della storia dall'Olimpo delle idealità alla prosaica realtà degli individui è finalizzata coscientemente all'indagine di una legge che è metastorica ed universale: la natura formale (e dunque prettamente linguistica) dei cambiamenti, ${ }^{19}$ che tiene celata l'immutabilità del sistema sociale e ne facilita la conservazione.

Verità e menzogna, come vantaggio e svantaggio, come progresso e regresso, come bene e male, sono termini indissolubili. E la più grande ed ultima verità sarebbe questa: che tutto è relativo; ma poiché il relativo non avrebbe senso se non s'opponesse all'assoluto, anche ciò è vero-fino ad un certo punto. ( $L$ 'Amore 515$)^{20}$

Impostando la propria narrativa intorno a queste tesi, De Roberto si poneva sulla via dell'effettivo superamento della visione verista, pur senza compieme l'intero cammino; ciò che gli impedì di procedere oltre i limiti di una transitoria originalità fu l'incapacità di riconoscere l'importanza che assume la forma della realtà (il punto di vista) se le conseguenze di ogni variazione di forma hanno davvero tanto peso.

\section{University of Toronto}

\section{NOTE}

1 Lo studio di De Roberto e l'archiviazione delle sue opere maggiori su supporto magnetico sono stati l'oggetlo della mia tesi universitaria, conclusa nel 1989 a Firenze, sotto la guida di Lanfranco Caretti e di Roberto Fedi. Il presente lavoro offre, nel loro sviluppo, alcune idee maturate allora sulla base di un archivio digitale dell'opera di De Roberto, considerevole per entità (quattro romanzi ed una trentina di novelle), filologicamente più corretto delle edizioni correnti, ma soprattutto fornito di un'estrema flessibilità di impiego, grazie 
agli strumenti di ricerca e di analisi ed ai criteri approntati durante il lavoro.

2 Per esteso, il nome della famiglia accompagnato dai titoli nobiliari, è: Uzeda, principi di Francalanza e Mirabella (1.2.3: 461).

3 Le citazioni, ove non sia specificata la provenienza, si intendono riferite ai Viceré. Il numero della pagina è preceduto da tre numeri separati dal punto, che si riferiscono, rispettivamente, alla parte, al capitolo e al paragrafo di appartenenza. Quando si cita L'Imperio, oltre al titolo si riportano il capitolo e la pagina.

4 Per chiudere il discorso menzionato di sopra, mi pare di vedere qui una conferma della presenza dell'autore appena celata dietro le riflessioni del passo precedente, il quale, in omaggio ai precetti della scuola verista, si sforza di riprodurre il pensiero del principe Giacomo e tuttavia vela debolmente l'intervento del narratore a condanna della natura tutta esteriore dell'istituzione nobiliare. Se risultava quasi grottesco (forse intenzionalmente) quel farsi vanto di mobili e livree in forza della loro "vecchiaia"-termine già negativoanziché della loro "antichità", l'osservazione di Giacomo circa il guardaportone, oramaj divenuto lusso per piccoli parvenu, è perfino contraddetta dall'opinione del figlio Consalvo che, a distanza di diversi capitoli, e quindi di anni (ne passano almeno dieci), esprime un senso vivo di orgoglio per il guardaportone (ora addirittura "maestoso"), mentre, ai suoi occhi, le livree ritomano addirittura "fiammanti" (come del resto erano state definite in un altro passo, 2.2.2: 729, nel giudizio dei popolani 'clienti' dello zio Gaspare, duca e deputato del regno), in un soprassalto di coerenza narrativa con il punto di vista dei personaggi.

5 Cfr. 3.3.3: 949, un passo dove si dichiara l'equivalenza sostanziale-mutata la forma-fra passato e presente, unificati dalla legge naturale che impone la prevaricazione del più forte, del più abile: "Come un tempo aveva gettato sulla folla il suo tiro a quattro, così la schiacciava adesso col peso della sua dottrina, e la gente che si tirava da canto, un tempo, per non restar sotto i suoi cavalli, esclamando tuttavia: «Che bell'equipaggio!» adesso lo stava a udire, intronata della sua loquela, dicendo: "Quante cose sa!»". D'altronde, lo stesso paragone tra la foga oratoria e la carrozza lanciata sulla folla non fa altro che accentuare la sopravvivenza di abitudini invalse da secoli nella stirpe dei viceré, poiché si richiama puntualmente ad un aneddoto riferito da donna Ferdinanda a Consalvo bambino: "Una volta che il capitano di giustizia con la carrozza propria ardì passar innanzi alla sua, sai che fece mio nonno? Lo aspettò al ritomo, ordinò al cocchiere di buttargli addosso i cavalli, gli fracassò il legno e gli pestò le costole!..." (1.4.4: 549).

6 Meditata è la scelta del personaggio di don Eugenio a rappresentare la figura del nobile in questo umoristico quadretto. All'introversione fantasticante e all'attivismo sterile si unisce in Eugenio la spocchia nobiliare, l'illusione del potere che gli dovrebbe derivare semplicemente dal sangue nobiliare e dal retaggio del passato: è proprio lui, pur velleitariamente, che cerca di tenere alta la dignità del casato, di difenderla dalle reiterate offese che scaturiscono dalla condotta dei familiari e della societa. D'altra parte don Eugenio, Ferdinando e suor Crocifissa rappresentano i falliti, coloro che sono effettivamente destinati a soccombere nella lotta per la sopravvivenza storica e sociale della nobiltà, incapaci di confrontarsi con quella realtà che i predecessori ed $\mathrm{i}$ parenti hanno saputo padroneggiare e che a loro resta pressoché ignota, confusa ai sogni di gloria e di evasione che perseguono invano.

7 Su tale aspetto si appunta l'ironia dell'autore, sul finire del romanzo, quando allo sprofondare di don Eugenio nella miseria e nella demenza senile corrisponde una comica enfatizzazione dei suoi titoli, in un catalogo fantastico per geografia ed onorificenze: "«Eugenio Consalvo Filippo Blasco Ferrante Francesco Maria Uzeda di Francalanza, Mirabella, Oragua, Lumera, etc., etc., Gentiluomo di Camera (con esercizio) di Sua Maestà, quello era re!» e si cavava il cappello «Ferdinando II; medagliato da Sua Altezza il Bey di Tunisi del Nisciam-Ifitkar, presidente dell'Accademia dei Quattro Poeti, membro corri- 
spondente di più società scientifico-letterario-vulcanologiche di Napoli, Londra, Parigi, Caropepe, Pietroburgo, Paoloburgo, Nuova York e Forlimpopoli, autore della celebre opera storico-araldico-nobilo-blasonico-gentilesco-cronologica intitolata l'Araldo Sicolo con supplimento... Un soldo, per comprarmi un sigaro...»" (3.6.3: 1009).

8 La natura meramente nominale degli ideali professati da Consalvo in politica è ribadita nell'Imperio: "Consalvo era divenuto democratico e progressista, promettendo di sedere a sinistra, di dare perfino una mano ai socialisti. Tutto questo non gli era costato nulla, o ben poco: parole, parole, parole" (2: 1144).

9 Ecco un altro esempio dello stesso procedimento, in un passo che irride al patriottismo del duca Gaspare: "nessuno pensava a prendere un provvedimento che dimostrasse al popolo come i tempi fossero cangiati e i privilegi distrutti e tutti i cittadini veramente ed assolutamente uguali. Egli propose e fece decretare l'abolizione del pane sopraffino" (1.8.2: 663).

10 Sul pessimismo di De Roberto, e sulla sua particolare natura, cfr. Tedesco 75-77, 127.

11 Non è fattore trascurabile la genesi milanese del romanzo, sorretta dalla frequentazione di artisti come Boito, Camerana, Gualdo, Giacosa, Rovetta, Praga e Oliva.

12 La singolarità della posizione dei viceré fra la nobiltà la ritroviamo accennata durante il racconto dell'infanzia e della formazione di Consalvo: "quelli, non potendogli rendere pane per focaccia, giacché il nomignolo degli Uzeda, I Viceré, diceva la loro antica potenza, se lo mettevano sotto" (1.6.2: 595-96); e quindi in un passo dell'Imperio, dove si pone l'accento ancora una volta sulla "potenza" degli Uzeda, presentando come uno dei requisiti fondamentali per la comprensione del personaggio di Consalvo "la presunzione ereditaria degli Uzeda, più conosciuti, laggiù in Sicilia, col nomignolo di «Viceré», poiché vantavano parecchi viceré tra gli antenati e dei viceré serbavano ancora il fasto ed il prestigio" (L'Imperio 2: 1141).

$13 \mathrm{Su}$ tale aspetto del romanzo, presente, a nostro avviso, ma non preminente, si sono fermati gli studi critici. Grana, ad esempio, reputa la raffigurazione della vita pubblica da parte di De Roberto alla stregua di una appendice della vita privata degli Uzeda; in proposito, cfr. anche Pomilio.

14 Le ambizioni di Lodovico sono perfettamente assimilabili a quelle di Gaspare e Consalvo, e non solo per il peso della chiesa nella società: la ricerca del potere cui Lodovico tende, percorrendo la scala della gerarchia ecclesiastica (secondo i canoni stendhaliani de $l l$ rosso e il nero), è descritta come un'aspirazione alla regalità, al potere assoluto, e quindi come una manifestazione della "prepotenza" degli Uzeda: "Ai Benedettini, infatti, c'era un regno da conquistare: l'Abate era una potenza, aveva non so quanti titoli feudali, un patrimonio favoloso da amministrare: le antiche Costituzioni di Sicilia gli davano il diritto di sedere tra i Pari del regno!" (1.3.2: 478). Anche in un altro caso, il campo di azione del Padre benedettino, il convento di San Nicola, è associato, per ruolo ed importanza, alla nobiltà più alta, in mezzo alla quale si fa menzione, naturalmente, anche dei viceré: cfr. 1.6.3: 606 .

$15 \mathrm{Si}$ confronti, in merito a questo, una frase dell'Amore che, riallacciandosi alle teorie di Lombroso, classifica la stessa genialità nel campo della variazione 'mostruosa': "tutti i genii sono il prodotto di anomalie della psiche" (353).

16 "Come in matematica, così, anzi più spesso in filosofia vi sono problemi che ammettono più d'una soluzione" (L'Amore 17).

17 Cfr. L'Amore 15: "non c'è né progresso né regresso in senso assoluto, ma diversità e complicazione".

18 Si confrontino, sul tema, le tesi riportate nell'Amore: "il banchiere che tenta una speculazione dice che essa ha per oggetto il bene del paese, il miglioramento delle industrie, l'incremento del commercio: ma fin dal primo istante egli ha piena coscienza ed esatta percezione della reale utilità sua propria. L'assoluto disinteresse, per tanto, non esiste, e 
tutte le nostre azioni sono suggerite da qualche interesse" (I05).

19 In forma più generale il romanzo dimostra la tesi che l'oggettività storica si impone come il prodotto non degli avvenimenti, bensì delle parole: i manifesti anonimi e gli articoli diffamatori che perseguitano Gaspare e Consalvo, riportando a galla momenti contraddittori della loro carriera politica, contengono pettegolezzi per lo più esatti, ma che vengono smentiti dalla loro forma calunniosa, mentre riceve credito la biografia del principino scritta da un agrimensore nello stile altisonante e letterariamente più dignitoso dell'agiografia. A dimostrazione del ruolo particolare ricoperto dal linguaggio e dalla letteratura possiamo ricordare che proprio in seguito alla lettura della biografia della Beata Ximena, sua illustre antenata, Teresina si persuade ad abbandonare la ricerca di un amore romantico, avendo scoperto un modello di comportamento (il sacrificio) che appaga le sue ambizioni eroiche e riveste con espressioni altisonanti una realtà avvilente, il matrimonio con il grossolano Michele.

20 Già De Sanctis aveva indicato simili conseguenze filosofiche derivanti dall'approfondimento delle teorie di Darwin, nella celebre conferenza romana sul "Darwinismo nell'arte", del 1883: "Questa maniera di concepire la vita ha indebolito in noi il senso del fisso e dell'assoluto. Collocandoci in un ambiente di continua trasformazione, concepiamo le cose nel loro divenire, in relazione con le loro origini e con l'ambiente ove sono nate; si è sviluppato in noi energicamente il senso del relativo. Il senso del reale, della forza e del relativo è il carattere della nostra trasformazione" (1103).

\section{OPERE CITATE}

Amheim, Rudolf. Entropia e arte. Saggio sul disordine e l' ordine. Torino: Einaudi, 1974.

Darwin, Charles. L'origine delle specie. Roma: Newton Compton, 1985.

De Roberto, Federico. I Viceré. In Romanzi novelle e saggi. Ed. Carlo A. Madrignani. Milano: Mondadori, 1984.

L'Imperio. In Romanzi novelle e saggi. Ed. Carlo A. Madrignani. Milano: Mondadori, 1984.

. L'Amore. Fisiologia. Psicologia. Morale. Milano: Galli, 1895.

Grana, Gianni. I Viceré e la patologia del reale. Discussione e analisi storica delle strutture del romanzo. Milano: Marzorati, 1982.

De Sanctis, Francesco. "Il darwinismo nell'arte". Scritti critici. Ed. Gianni Scalia. Milano: Rizzoli, 1966. 1093-111.

Di Grado, Antonio. Federico De Roberto e la "scuola antropologica". Positivismo, verismo, leopardismo. Bologna: Pàtron, 1982.

Navarria, Aurelio. Federico De Roberto. La vita e l'opera. Catania: Giannotta, 1974.

Pomilio, Mario. "L'antirisorgimento di De Roberto". Le ragioni narrative 1.6 (1960).

Tedesco, Natale. La norma del negativo. De Roberto e il realismo analitico. Palermo: Sellerio, 1981. 